\title{
Computational Simulation of Tumor Surgical Resection Coupled with the Immune System Response to Neoplastic Cells
}

\author{
J. Jesús Naveja, ${ }^{1}$ Flavio F. Contreras-Torres, ${ }^{1,2}$ \\ Andrés Rodríguez-Galván, ${ }^{1}$ and Erick Martínez-Lorán ${ }^{1}$ \\ ${ }^{1}$ Technology and Consultancy, CTDAT, 04360 Mexico City, Mexico \\ ${ }^{2}$ Universidad de Investigación de Tecnología Experimental Yachay, 100119 Urcuqui, Ecuador \\ Correspondence should be addressed to J. Jesús Naveja; naveja.jesus@ctdat.com
}

Received 14 September 2014; Revised 12 November 2014; Accepted 12 November 2014; Published 31 December 2014

Academic Editor: Gabriela M. Wilson

Copyright (C) 2014 J. Jesús Naveja et al. This is an open access article distributed under the Creative Commons Attribution License, which permits unrestricted use, distribution, and reproduction in any medium, provided the original work is properly cited.

Numerous mathematical and computational models have arisen to study and predict the effects of diverse therapies against cancer (e.g., chemotherapy, immunotherapy, and even therapies under research with oncolytic viruses) but, unfortunately, few efforts have been directed towards development of tumor resection models, the first therapy against cancer. The model hereby presented was stated upon fundamental assumptions to produce a predictor of the clinical outcomes of patients undergoing a tumor resection. It uses ordinary differential equations validated for predicting the immune system response and the tumor growth in oncologic patients. This model could be further extended to a personalized prognosis predictor and tools for improving therapeutic strategies.

\section{Introduction}

The most recent mathematical models are relegating Gompertzian growth curves out of tumor growth modeling. Gompertzian growth strongly depends on time [1], and it can be demonstrated that this leads to artifacts in tumor growth models working with external perturbations, that is, any given therapy. Thus, ordinary differential equations (ODEs) that resemble more the behavior of perturbed tumors [2-4] also share more similarities with the ODEs from the LotkaVolterra predator-prey model and with the logistic curve described by Verhulst. These ODEs are less time-dependent and focus on interactions among different populations and the carrying capacity of the system [5]. Other approaches for modeling tumor growth use complexity models $[6,7]$ or physically based models $[8,9]$, although they have been applied less on therapeutic models than ODEs.

Metastasis, the spread of the malignant cells through the body, causes the degeneration of different body functions, depending on the systems affected. Some of the computational models for metastasis are (a) those belonging to the field of complexity - where discrete models based on single cell interactions have been developed [10] —and (b) models with ODEs [11, 12]. These models are useful for predicting outcomes for patients under antimetastatic drugs therapies.

Another important factor affecting the dynamics of the tumor is the immune system. De Pillis et al. developed a model that includes as input some features of natural killer cells (NK) and T CD8+ cells (TCD8), as well as the tumor growth rate. NK and TCD8 cells mediate most of the immune response against tumor growth. This model was incorporated into another one that was able to predict clinical outcomes in patients receiving immunotherapy. Interestingly, this model neglects metastasis but obtains clinically significant data [13].

Tumor resection, although a traditional treatment for cancer that is still applied as a treatment for some types of cancer, is a therapy whose mathematical modeling has not been fully developed. Remarkably, a biophysical model correlates histopathology findings with nutrient diffusion of tumor cells, thus permitting a prediction of the required tumor surgical resection for enhancing the probability of total remission [14]. Also, some studies in the field of probability predict the necessity of radiotherapy in postsurgical oncologic patients [15]. However, there are no models with ODEs or in the field of complexity, even though much clinical data have been recollected about tumor resection. It is well known that 
many tumor surgeries lead to recurrence, and the most accepted mechanism for this is that resections are not always complete, so that negative surgical margins are critical for ensuring a better resection [16]. Also, deliberated partial resections are common, particularly when the tumor is located in organs whose function's maintenance is necessary (e.g., kidney, brain) [16, 17].

In this study, De Pillis et al. ordinary differential equations were coupled with a computational model of tumor resection to observe if the response of the immune system could be modified by a resection of a massive percentage of the tumor. This is a novel study using ODEs for modeling the immune system and tumor partial resection as perturbations on a tumor.

\section{Methodology}

Using Python 2.7 a program was developed to integrate the ODEs describing the immune system, the tumor, and their interactions. From De Pillis et al.,

$$
\begin{aligned}
& \frac{d T}{d t}=a T(1-b T)-c N T-D, \\
& \frac{d N}{d t}=\sigma-f N+\frac{g T^{2}}{h+T^{2}} N-p N T, \\
& \frac{d L}{d t}=-m L+\frac{j D^{2}}{k+D^{2}} L-q L T+r N T,
\end{aligned}
$$

where

$$
D=d \frac{(L / T)^{\lambda}}{s+(L / T)^{\lambda}} T
$$

where $t$ is time, $T$ is the number of tumor cells, $N$ is the number of NK cells, $L$ is the number of TCD8 cells, and $D$ is the fractional cell kill. The other terms are constants describing the characteristics of the immune system and tumor cells and are better defined in the article from the group of De Pillis, although a brief description of them is given in Table 1.

The parameters that predict whether the immune systems response is either competent (i.e., an immune system capable of defeating a malignant tumor) or incompetent (i.e., the opposite, an immune system whose response is not sufficient and would be overwhelmed by a malignant tumor) against the tumor growth were extracted from the same reference and are given in Table 1.

The incompetent immune system was perturbed during tumor growth with a sudden removal of tumor cells at an arbitrary time. It was then observed whether the immune response remained insufficient against the tumor. The time of the surgery and its extent were deliberately changed in different simulations. Thus, the surgery is evidently an algorithm, not an ODE in this model.

\section{Results}

The data from De Pillis et al. for competent and incompetent immune system responses against tumors were reproduced.

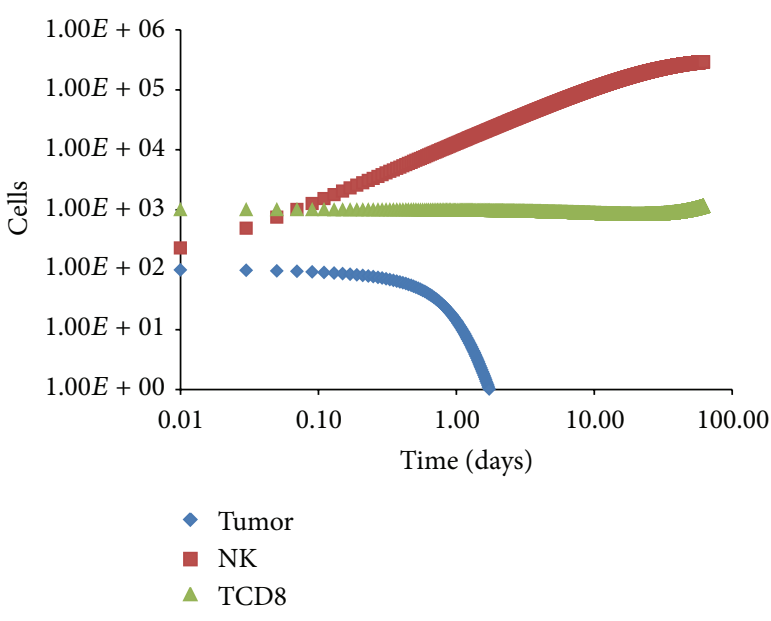

FIGURE 1: Competent immune system versus tumor. NK cell number increase of about 3 orders of magnitude. TCD 8 cell increment is less marked, but necessary for an efficient response.

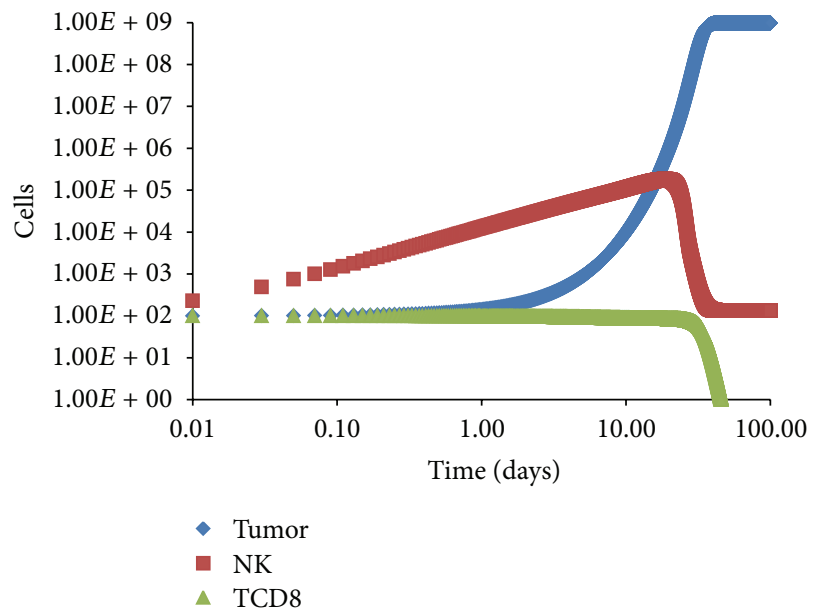

FigURE 2: Incompetent immune system overwhelmed by a tumor.

The parameters used for the simulations are described in Table 1. When the immune system is overwhelmed by the tumor, a marked decrement of its response is observed, an effect that does not happen in competent immune systems, as shown in Figures 1 and 2. In Figure 1, NK cells exhibit a rapid growth that leads to tumor extinction; interestingly, TCD8 cells number is not modified at a large extent. Nonetheless, TCD8 cell stability is essential for attacking the tumor, as may be seen in Figure 2, where tumor growth elicits a rapid response, at first primarily dependent on NK cells, but with entire collapse of the immune system occurring at around day 20.

Remarkably, when the incompetent immune system is perturbed with a partial but important surgery, an inflection in the remaining tumor cells is observed with practically the same immune response, which does not modify (Figure 3). However, the immune system collapse observed in Figure 2 is not reproduced when the surgery is performed. The key 
TAble 1: Parameters defining a competent and an incompetent immune system for a given tumor (modified from [13]).

\begin{tabular}{|c|c|c|c|c|}
\hline Parameter & Units & Meaning & Competent & Incompetent \\
\hline$a$ & Day $^{-1}$ & Tumor growth rate & $5.14 \times 10^{-1}$ & $5.14 \times 10^{-1}$ \\
\hline$b$ & Cell $^{-1}$ & $1 / b$ is tumor carrying capacity & $1.02 \times 10^{-9}$ & $1.02 \times 10^{-9}$ \\
\hline$c$ & Cell $^{-1}$ day $^{-1}$ & Fractional tumor cell kill by NK cells & $3.23 \times 10^{-7}$ & $3.23 \times 10^{-7}$ \\
\hline$d$ & Day $^{-1}$ & Saturation level of fractional tumor cell kill by TCD8 cells & 5.8 & 5.8 \\
\hline$\lambda$ & None & Exponent of fractional tumor cell kill by TCD8 cells & 1.36 & 1.36 \\
\hline$s$ & None & Steepness coefficient of the tumor-TCD8 cells competition & 2.5 & 2.5 \\
\hline$\sigma$ & Cells day ${ }^{-1}$ & Constant source of NK cells & $1.3 \times 10^{4}$ & $1.3 \times 10^{4}$ \\
\hline$f$ & Day $^{-1}$ & Death rate of NK cells & $4.12 \times 10^{-2}$ & $4.12 \times 10^{-2}$ \\
\hline$g$ & Day $^{-1}$ & Maximum NK cell recruitment rate by tumor cells & $2.5 \times 10^{-2}$ & $2.5 \times 10^{-2}$ \\
\hline$h$ & Cell $^{2}$ & Steepness coefficient of the NK cell recruitment curve & $2.02 \times 10^{7}$ & $2.02 \times 10^{7}$ \\
\hline$p$ & Cell $^{-1}$ day $^{-1}$ & NK cell inactivation rate by tumor cells & $1.0 \times 10^{-7}$ & $1.0 \times 10^{-7}$ \\
\hline$m$ & Day $^{-1}$ & Death rate of TCD8 cells & $2.0 \times 10^{-2}$ & $2.0 \times 10^{-2}$ \\
\hline j & Day $^{-1}$ & Maximum TCD8 cell recruitment rate & $3.75 \times 10^{-2}$ & $3.75 \times 10^{-2}$ \\
\hline$k$ & Cell $^{2}$ & Steepness coefficient of the TCD8 cell recruitment curve & $2.0 \times 10^{7}$ & $2.0 \times 10^{7}$ \\
\hline$q$ & Cell $^{-1}$ day $^{-1}$ & TCD8 cell inactivation rate by tumor cells & $3.42 \times 10^{-10}$ & $3.42 \times 10^{-10}$ \\
\hline$r$ & Cell $^{-1}$ day $^{-1}$ & $\begin{array}{l}\text { Rate at which tumor-specific TCD } 8 \text { cells are stimulated to be } \\
\text { produced as a result of tumor cells killed by NK cells }\end{array}$ & $1.1 \times 10^{-7}$ & $1.1 \times 10^{-7}$ \\
\hline$T(0)$ & Cells & Initial number of tumor cells & 100 & 100 \\
\hline$N(0)$ & Cells & Initial number of NK cells & 200 & 200 \\
\hline$L(0)$ & Cells & Initial number of TCD8 cells & 1000 & 100 \\
\hline
\end{tabular}

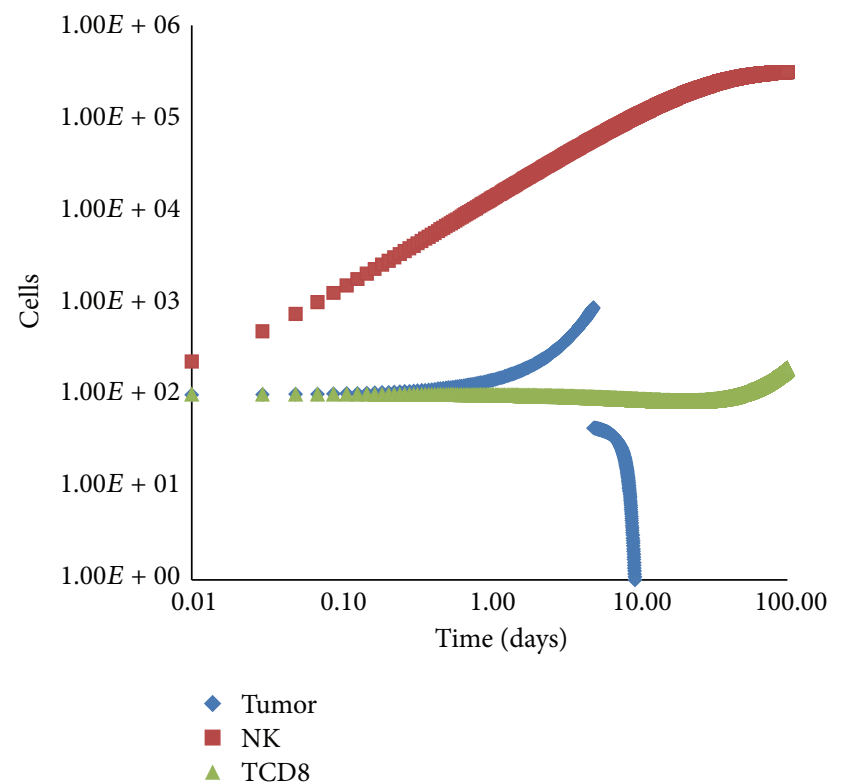

FIGURE 3: The same incompetent immune system depicted in Figure 2, plus a 95 percent resection at day 5; an inflection in the tumor growth curve is seen after the surgery.

finding is that this more vulnerable system is capable of reducing the remaining tumor cells.

In order to recognize the extent and the time of the surgery as important variables when a tumor surgical resection is performed, different percentages of tumor removal and

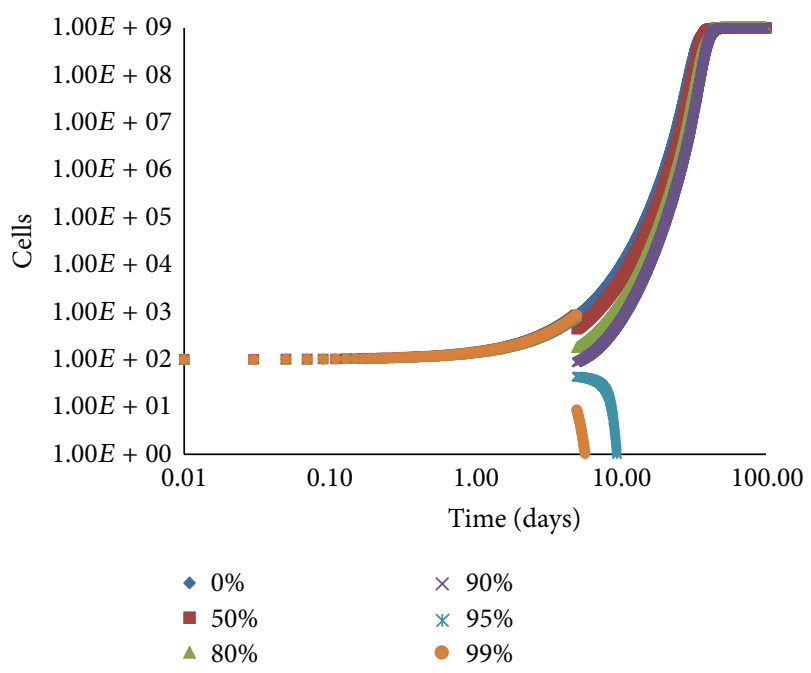

FIgURE 4: Tumor growth resulting from different percentages of tumor resection: $0,50,80,90,95$, and $99 \%$ of the tumor, but with the same immune system as in Figure 2 and all of them at day 5.

times for surgery were tested (Figures 4 and 5, resp.). At day 5 , and for the same immune system response seen in Figure 2, only surgeries that remove $95 \%$ or more of the tumor would cure the patient (assuming that no other intervention is performed) (Figure 4). On the other hand, a 95\% resection would only cure the patient if it is performed earlier than day 10 (Figure 5). 


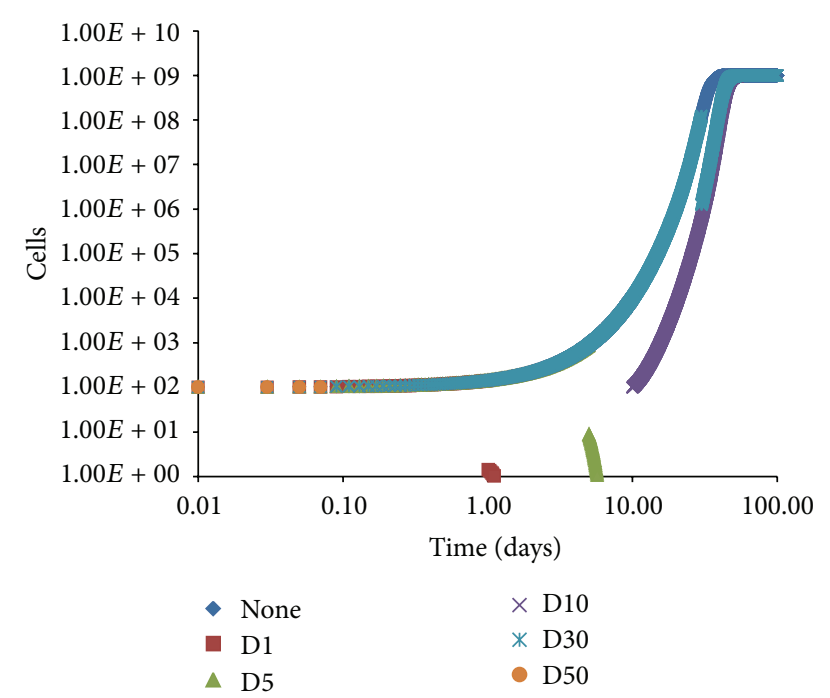

FIgURE 5: Tumor growth for resections at days 1, 5, 10, 30, and 50; the extent of the surgery was set at $95 \%$ and the immune system is the same as that shown in Figure 2.

\section{Discussion}

A decline in tumor growth could not be elicited by the immune system in every case. In Figure 1, a sufficient immune response performed by a competent immune system leads to the inhibition of the tumor. However, a partial but sufficient surgery may reverse the overwhelming of the immune system response when otherwise it would be incompetent (e.g., Figures 2 and 3). When the surgery was implemented, the most important variables turned out to be (a) the tumor size at the time of the surgery, which is a variable dependent on the time of the surgery, as can be seen in Figure 5, where only resections performed before tumor growth reaches a significant inflexion point are curative; (b) the extent of the surgery: Figure 4 shows how resections reducing the number of tumor cells below a critical number (50 cells for this particular tumor interacting with this particular immune system) may lead to total remission; and (c) the immune system response at the time of the surgery, which was found to be equivalent to the number of cells in the immune system, which only defines a higher or lower critical tumor mass (data not shown). Interestingly, clinical data from a study with resection of different percentages of the tumor highly resembled this data: only resections spanning $90 \%$ or more of the tumor volume were likely to lead to a total remission of abdominal Burkitt's lymphoma; this can be seen in Figure 4 [18]. It must be noted that in the simulation depicted in Figure 5 the critical number of cells (a threshold that, if trespassed, will lead to a massive growth of the tumor) is consistent with that in Figure 4. This effect shows that the time of resection is not by itself a prognostic factor.

The assumptions taken by this model define its limitations. It does not take into account metastasis, adjuvant chemotherapy, or tumor angiogenesis. Nonetheless, studying a single variable in this case would be more reliable. Thus, this model would work better for predicting the behavior of tumors in which angiogenesis and metastasis may be neglected. This is an evident limitation when predicting outcomes distant in time. Hence, this model would work better for predicting clinical events foreseen to occur soon when considering tumors that metastasize, since the possibility of distant metastasis increases with time. Although the timing of the interventions in this model is early, it must be taken into account that the patients from [13] had an advanced disease. This does not diminish the reliability of the model, as it may be personalized to every patient. On the other hand, it could be difficult to estimate the proportion of the tumor extracted. This problem may be avoided since a correlation exists between the volume of the tumor and the number of cells in the tumor, and computed tomography can estimate tumor volume accurately [19]. Diffusion-tensor magnetic resonance may even estimate brain tumor cellularity precisely [20].

This study was performed on a validated model of immune system against tumor growth and could be further developed to consider metastasis, chemotherapy, and any other therapy for every individual case. This will bring a more personalized health care and prognosis depending on more than population statistic variables, as in the prevalent prognosis calculation [21]. Besides this, it would be highly beneficial if surgeons could be aided with computer programs for improving the patients' follow-up and scheduling subsequent surgeries based on evidence.

\section{Conclusion}

A novel computational model is hereby proposed, which predicts the interaction of tumors and immune system response and their behavior after a partial surgical resection using ordinary differential equations. It was shown that a partial but sufficient surgery could help the immune system defeat a tumor in silico. Otherwise, the immune system explored would have collapsed and been incapable of eradicating the neoplasm.

Further research is needed in order to improve prognostic tools and therapeutic strategies using computational models predicting clinical outcomes of patients with cancer. This computational model requires clinical validation, although it is based on a model of immune system that correlated well with the outcomes of patients with cancer. Moreover, ODEs should be tested, for example, against stochastic differential equations, in order to assess their reliability.

\section{Conflict of Interests}

The authors declare that there is no conflict of interests regarding the publication of this paper.

\section{Acknowledgments}

The authors thank Ricardo Espinosa-González for his contribution revising the paper and for his pertinent annotations. J. Jesús Naveja acknowledges PECEM M.D./Ph.D. program from the Faculty of Medicine of UNAM for the organization of the fellowship. Flavio F. Contreras-Torres thanks Yachay Tech University for supporting his academic stay. 


\section{References}

[1] A. K. Laird, "Dynamics of tumor growth," British Journal of Cancer, vol. 18, no. 3, pp. 490-502, 1964.

[2] N. L. Komarova and D. Wodarz, "ODE models for oncolytic virus dynamics," Journal of Theoretical Biology, vol. 263, no. 4, pp. 530-543, 2010.

[3] R. K. Sachs, L. R. Hlatky, and P. Hahnfeldt, "Simple ODE models of tumor growth and anti-angiogenic or radiation treatment," Mathematical and Computer Modelling, vol. 33, no. 12-13, pp. 1297-1305, 2001.

[4] E. Comen, P. G. Morris, and L. Norton, "Translating mathematical modeling of tumor growth patterns into novel therapeutic approaches for breast cancer," Journal of Mammary Gland Biology and Neoplasia, vol. 17, no. 3-4, pp. 241-249, 2012.

[5] A. A. Berryman, "The origins and evolution of predator-prey theory," Ecology, vol. 73, no. 5, pp. 1530-1535, 1992.

[6] C. F. Lo, "Stochastic Gompertz model of tumour cell growth," Journal of Theoretical Biology, vol. 248, no. 2, pp. 317-321, 2007.

[7] A. R. Kansal, S. Torquato, G. R. Harsh IV, E. A. Chiocca, and T. S. Deisboeck, "Simulated brain tumor growth dynamics using a three-dimensional cellular automaton," Journal of Theoretical Biology, vol. 203, no. 4, pp. 367-382, 2000.

[8] T. Yamano, "Statistical ensemble theory of Gompertz growth model," Entropy, vol. 11, no. 4, pp. 807-819, 2009.

[9] P. Castorina and D. Zappalà, “Tumor Gompertzian growth by cellular energetic balance," Physica Acta, vol. 365, no. 2, pp. 473480, 2006.

[10] L. A. Liotta, G. M. Saidel, and J. Kleinerman, "Stochastic model of metastases formation," Biometrics. Journal of the Biometric Society, vol. 32, no. 3, pp. 535-550, 1976.

[11] V. Haustein and U. Schumacher, "A dynamic model for tumour growth and metastasis formation," Journal of Clinical Bioinformatics, vol. 2, no. 1, article 11, 2012.

[12] A. R. A. Anderson, M. A. J. Chaplain, E. L. Newman, R. J. C. Steele, and A. M. Thompson, "Mathematical modelling of tumour invasion and metastasis," Journal of Theoretical Medicine, vol. 2, no. 2, pp. 129-154, 2000.

[13] L. G. De Pillis, A. E. Radunskaya, and C. L. Wiseman, "A validated mathematical model of cell-mediated immune response to tumor growth," Cancer Research, vol. 65, no. 17, pp. 79507958, 2005.

[14] M. E. Edgerton, Y.-L. Chuang, P. MacKlin, W. Yang, E. L. Bearer, and V. Cristini, "A novel, patient-specific mathematical pathology approach for assessment of surgical volume: application to ductal carcinoma in situ of the breast," Analytical Cellular Pathology, vol. 34, no. 5, pp. 247-263, 2011.

[15] F. M. O. Al-Dweri, D. Guirado, A. M. Lallena, and V. Pedraza, "Effect on tumour control of time interval between surgery and postoperative radiotherapy: An empirical approach using Monte Carlo simulation," Physics in Medicine and Biology, vol. 49, no. 13, pp. 2827-2839, 2004.

[16] S. E. Sutherland, M. I. Resnick, G. T. Maclennan, and H. B. Goldman, "Does the size of the surgical margin in partial nephrectomy for renal cell cancer really matter?” The Journal of Urology, vol. 167, no. 1, pp. 61-64, 2002.

[17] I. Ciric, M. Ammirati, N. Vick, and M. Mikhael, "Supratentorial gliomas: surgical considerations and immediate postoperative results. Gross total resection versus partial resection," Neurosurgery, vol. 21, no. 1, pp. 21-26, 1987.
[18] I. T. Magrath, S. Lwanga, W. Carswell, and N. Harrison, "Surgical reduction of tumour bulk in management of abdominal Burkitt's lymphoma," British Medical Journal, vol. 2, no. 914, pp. 308-312, 1974.

[19] C. R. Johnson, H. D. Thames, D. T. Huang, and R. K. SchmidtUllrich, "The tumor volume and clonogen number relationship: tumor control predictions based upon tumor volume estimates derived from computed tomography," International Journal of Radiation Oncology, Biology, Physics, vol. 33, no. 2, pp. 281-287, 1995.

[20] K. M. Gauvain, R. C. McKinstry, P. Mukherjee et al., "Evaluating pediatric brain tumor cellularity with diffusion-tensor imaging," American Journal of Roentgenology, vol. 177, no. 2, pp. 449454, 2001.

[21] B. Gwilliam, V. Keeley, C. Todd et al., "Development of prognosis in palliative care study (PiPS) predictor models to improve prognostication in advanced cancer: prospective cohort study," British Medical Journal, vol. 343, no. 7821, Article ID d4920, 2011. 


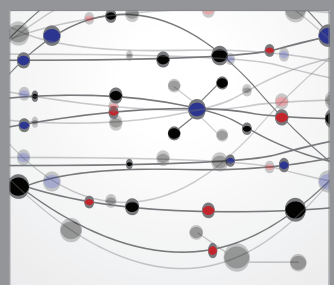

The Scientific World Journal
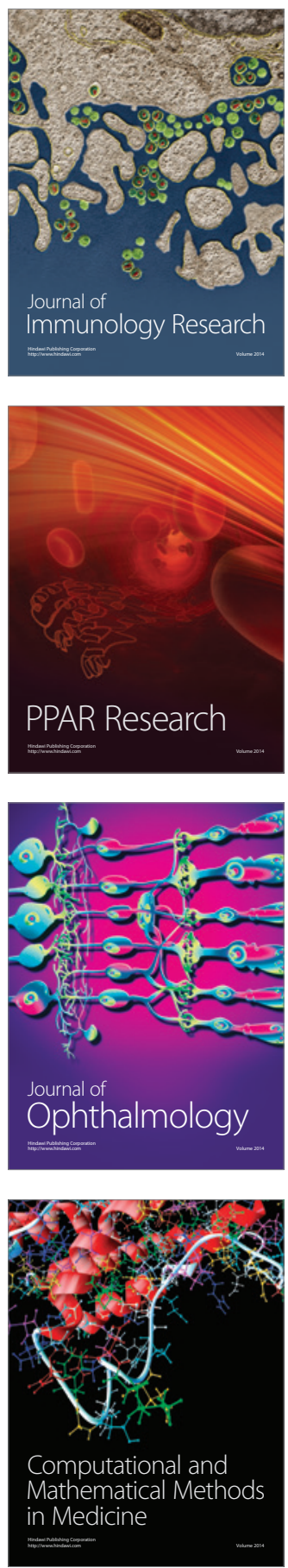

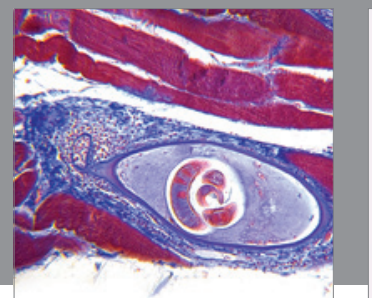

Gastroenterology

Research and Practice
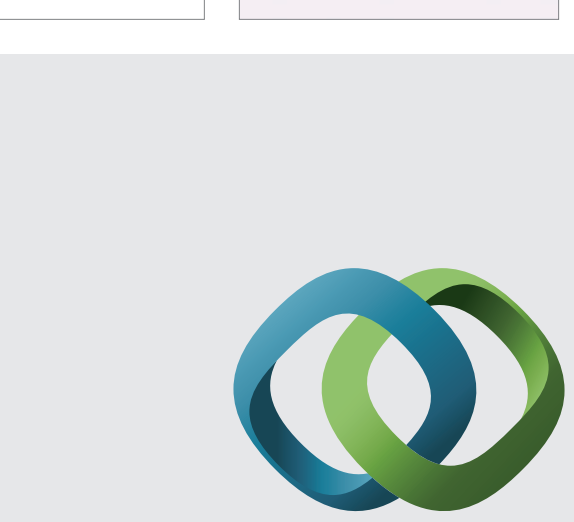

\section{Hindawi}

Submit your manuscripts at

http://www.hindawi.com
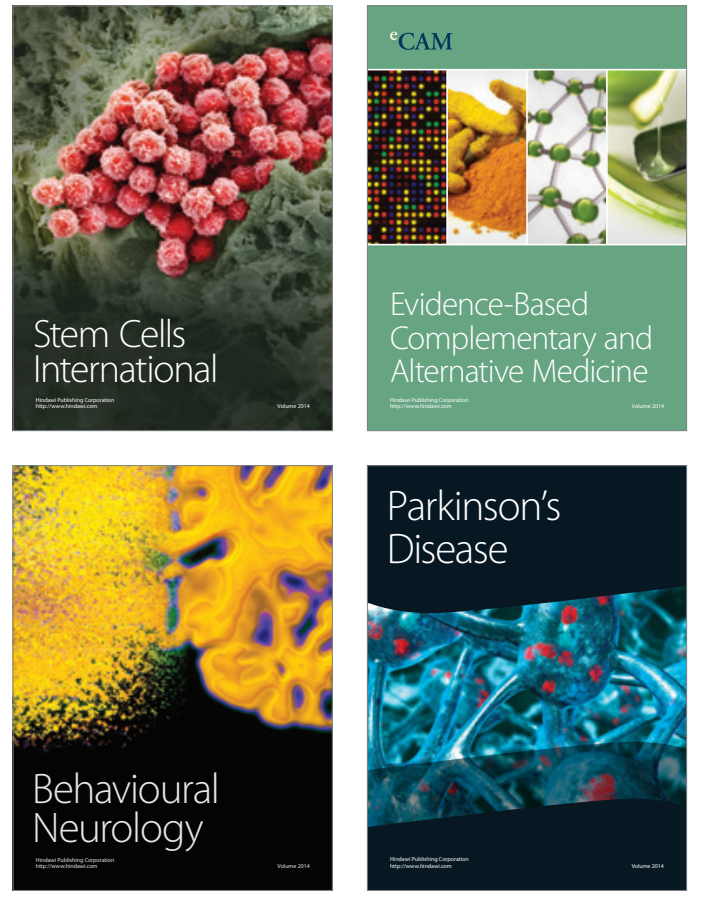
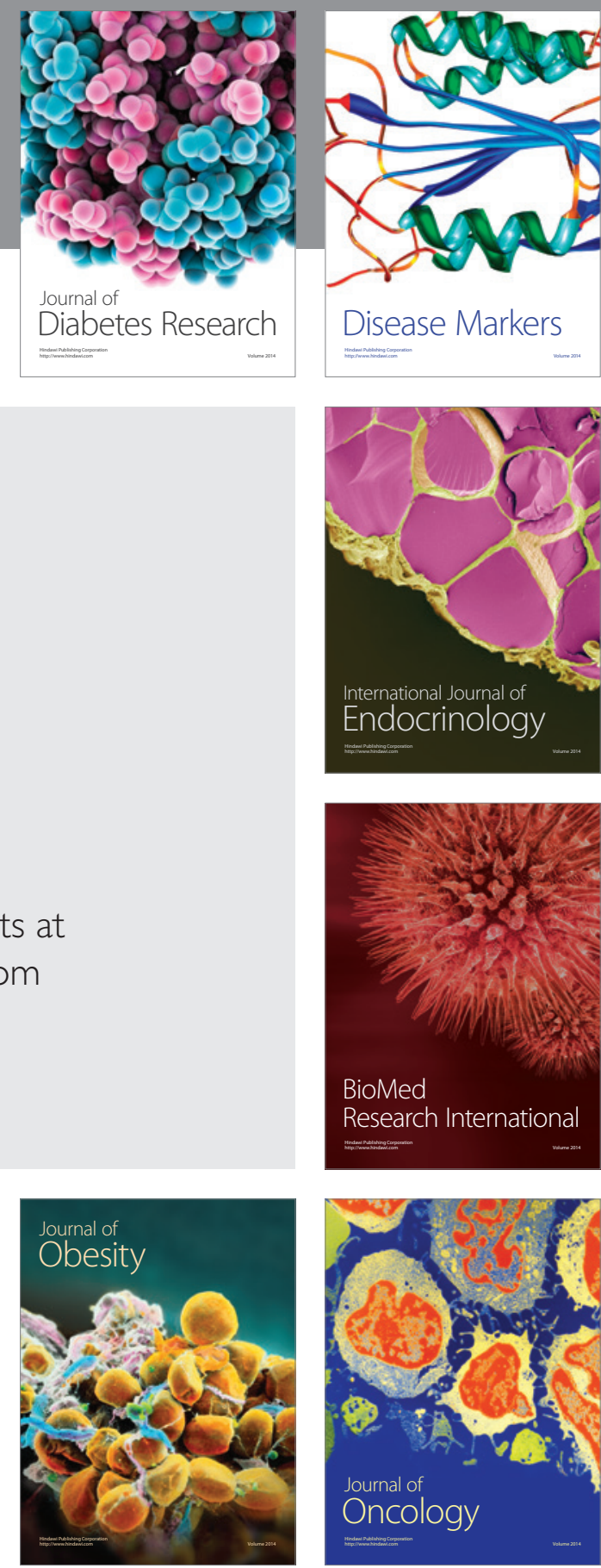

Disease Markers
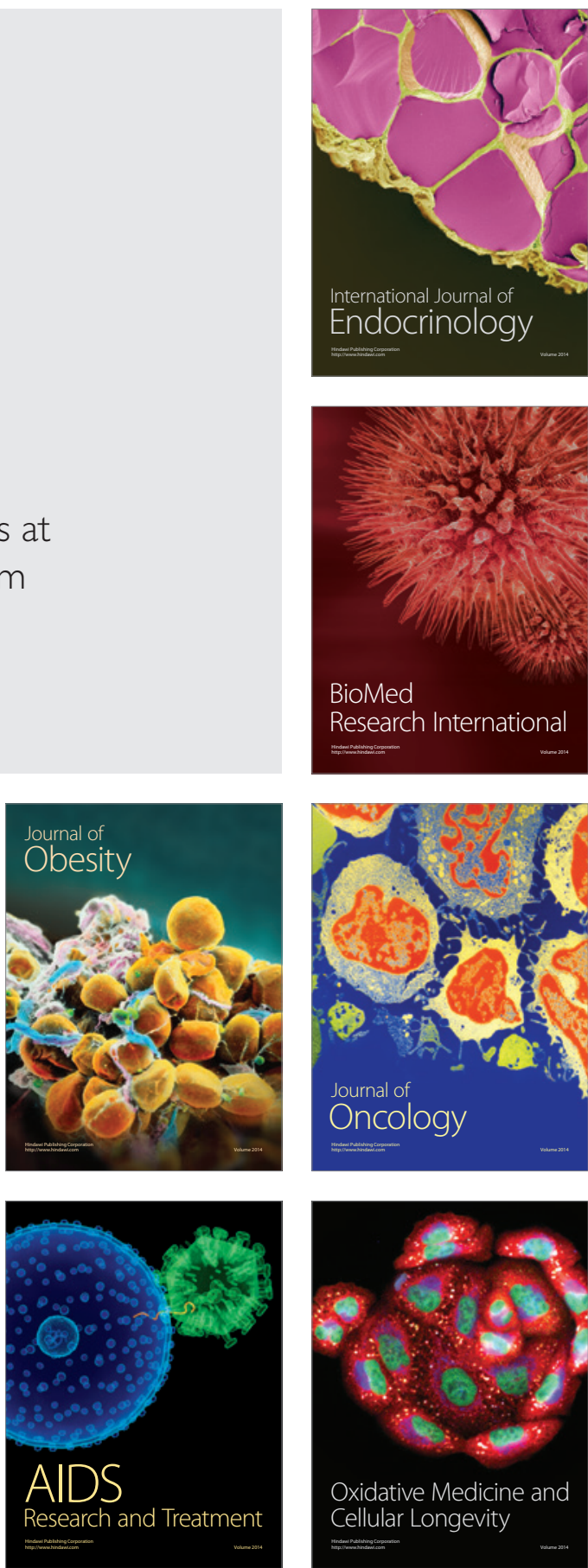